\title{
Pratiques discrètes de résistance des migrants roms à Turin et à Marseille
}

Discrete Practices of Resistance by Roma Migrants in Turin and Marseille

\section{Elisabetta Rosa}

\section{(2) OpenEdition \\ 1 Journals}

\section{Édition électronique}

URL : http://journals.openedition.org/conflits/19133

DOI : $10.4000 /$ conflits. 19133

ISSN : $1777-5345$

Éditeur :

CCLS - Centre d'études sur les conflits lilberté et sécurité, L'Harmattan

\section{Édition imprimée}

Date de publication : 19 mai 2016

Pagination : 19-34

ISBN : 978-2-343-09459-5

ISSN : 1157-996X

Référence électronique

Elisabetta Rosa, "Pratiques discrètes de résistance des migrants roms à Turin et à Marseille », Cultures \& Conflits [En ligne], 101 | printemps 2016, mis en ligne le 19 mai 2017, consulté le 30 mars 2021. URL : http://journals.openedition.org/conflits/19133; DOI : https://doi.org/10.4000/conflits. 19133 


\section{Pratiques discrètes de résistance des migrants roms à Turin et à Marseille}

\section{Elisabetta ROSA 1}

Elisabetta Rosa est urbaniste-géographe, post-doctorante à Aix-Marseille Université, CNRS, UMR 7303 Telemme, LabexMed. Ses recherches portent sur les marges urbaines et l'habitat précaire dans les villes d'Europe du sud et sur les pratiques spatiales de migrants "roms" en condition précaire. Elle a notamment publié "Marginality as resource? From Roma people territorial practices, an epistemological reframing of urban marginality ", in Lancione M. (dir.), Rethinking Life at the Margins, Londres, Routledge, 2016, pp. 182-197 et "Les marges et l'entredeux. Petite histoire d'une porte et des migrants "roms" en condition précaire ", in Canepari E., Mésini B., Mourlane S. (dir.), Mobil hom(m)es. Formes d'habitats et modes d'habiter la mobilité (XVIe-XXIe siècles), Ed. de L'aube, 2016, pp. 93-101.

\section{Roms migrants et résistance, un binôme complexe}

$\mathrm{D}$ ans l'introduction à l'ouvrage Geographies of Resistance, Steve Pile soutient qu' "il ne suffit plus de commencer des histoires de résistance avec des histoires de soi-disant pouvoir [...]. En revanche, les subjectivités politiques résistantes se constituent à travers des positions assumées non pas seulement en relation à l'autorité [...] mais aussi à travers des expériences qui ne peuvent pas être immédiatement qualifiées de "pouvoir", tels le désir et la rage, la capacité et l'habilité, le bonheur et la peur, le rêve et l'oubli 2 ». Si la

1. Ce travail a été réalisé dans le cadre du laboratoire d'excellence LabexMed-Les sciences humaines et sociales au cœur de l'interdisciplinarité pour la Méditerranée portant la référence 10-LABX-0090. Il a bénéficié d'une aide de l'État gérée par l'Agence nationale de la recherche au titre du projet Investissements d'Avenir $A *$ MIDEX portant la référence ${ }^{\circ}$ ANR-11IDEX-0001-02. En 2013, la recherche a été réalisée dans le cadre du programme postdoctoral Fernand Braudel-IFER-FMSH, soutenu par l'Union européenne (Programme Action Marie Curie - COFOUND - 7ème PCRD), $\mathrm{n}^{\circ}$ de subvention 245743. Elle a également été réalisée dans le cadre du programme ANR « Marges et villes entre exclusion et intégration. Cas méditerranéens».

2. "It is no longer enough to begin stories of resistance with stories of so-called power [...]. Instead, resistant political subjectivities are constituted through positions taken up not only in relation to authority [...] but also through experiences which are not so quickly labelled "power", such as desire and anger, capacity and ability, happiness and fear, dreaming and for- 
géographie critique s'est attachée, depuis longtemps, à analyser les processus de domination et les pratiques de résistance qui s'y opposent, ainsi que les rapports à l'espace des uns et des autres, il s'agit ici pour Pile de remettre en question le binôme pouvoir/résistance pour montrer, notamment, comment la résistance cherche à « occuper, développer et créer des spatialités alternatives à celles définies à travers l'oppression et l'exploitation ${ }^{3}$ ». C'est avec cet esprit que nous avons abordé le thème des pratiques de résistance des migrants roms en condition de précarité dans le cadre de nos récentes recherches portant sur l'habitat illégal et précaire à Marseille et à Turin.

Le squat, à savoir l'occupation sans droit ni titre d'un local, d'un bâtiment ou d'un terrain en vue de son habitation ou de son utilisation collective ${ }^{4}$, est souvent pratiqué et interprété comme alternative résistante au manque de logement, à la précarisation extrême de l'habitat, au dénuement, à la ségrégation, à la discrimination 5 . Parler des migrants roms et de leurs pratiques spatiales dans la ville permet d'aller plus loin dans cette réflexion. En effet, alors que les squats et les bidonvilles représentent souvent la seule possibilité pour ces populations de s'installer en ville, les questions de résistance sont très rarement mentionnées à ce propos. C'est plutôt le caractère "rom » qui est alors souligné, une labélisation ethnique qui obscurcit les diverses spécificités de ces pratiques et des espaces qui les soutiennent. L'ethnicisation portée par les discours et les politiques publiques conduit à occulter les différentes manières dont ces personnes habitent, accèdent ou revendiquent l'accès à la ville, ce qui perpétue leur ségrégation ${ }^{6}$. Conséquence des traitements que leur réservent les politiques et les pouvoirs publics, les Roms finissent par n'être que des objets passifs, aucune considération n'étant portée à leurs compétences, leurs capacités d'action et leur interaction avec le reste de la ville.

Des recherches récentes ont toutefois nuancé ces interprétations en mettant en avant le fait que les Roms n'attendent pas toujours après des politiques publiques pour satisfaire leurs besoins ${ }^{7}$. De plus, les mêmes politiques qui les marginalisent sont mises à l'épreuve par leurs pratiques de la ville et par la mobilisation de ressources qui en découle ${ }^{8}$.

getting ", Pile S., "Introduction », in Pile S., Keith M. (eds), Geographies of Resistance, Londres/New York, Routledge, 1997, p. 3 (notre traduction).

3 . «[...] occupy, deploy and create alternative spatialities from those defined through oppression and exploitation ", Pile S., "Introduction ", op. cit., p. 3 (notre traduction).

4. Péchu C., Les Squats, Paris, Presses de Sciences Po, 2010.

5. Pichon P., «Vivre sans domicile fixe : l'épreuve de l'habitat précaire », Communications, $\mathrm{n}^{\circ} 73,2002$, pp. 11-29. Bouillon F., « Des migrants et des squats : précarités et résistances aux marges de la ville ", Revue européenne des migrations internationales, vol. 19, n², 2003, pp. 23-46 (http://remi.revues.org/440, consulté le 26/06/2015). Péchu C., «Entre résistance et contestation. La genèse du squat comme mode d'action ", Travaux de science politique de l'Université de Lausanne, $\mathrm{n}^{\circ}$ 24, 2006, pp. 3-51.

6. Nacu A., «Un double langage : les usages du "politiquement correct” dans les programmes destinés aux Roms en Roumanie et en Bulgarie ", Études Tsiganes, n 38, 2010, pp. 86-106.

7. Legros O., «Les pouvoirs publics et la “question rom” en Europe aujourd'hui. Perspectives de recherche pour une approche comparative », Études tsiganes, n 39-40, 2009, pp. 42-55. 
Deux questions se posent alors très clairement : dans quels cas et en quels termes les pratiques spatiales des Roms sont, deviennent ou peuvent être interprétées comme des pratiques résistantes ? À quoi s'oppose cette résistance et envers qui s'exerce-t-elle ? Ces questionnements nous semblent fondamentaux pour (re)mettre les capacités d'action des Roms au centre de l'analyse et de la ville, sans pour autant que ce renversement de perspective aboutisse à une glorification de ces capacités et des personnes qui les portent ${ }^{9}$. Loin d'une telle interprétation, notre intention est plutôt de montrer comment un regard portant sur les pratiques permet de dépasser les oppositions dichotomiques entre pouvoir et résistance, dominants et subalternes. La résistance à la marginalité et à la précarité dont témoignent ces pratiques ne saurait être envisagée comme opposition claire et nette; il s'agit plutôt d'une mise à l'épreuve quotidienne qui se construit à travers les interactions entre les différents acteurs qui pratiquent la ville. Interpréter ces pratiques comme des "résistances discrètes ", postulat qui fonde notre hypothèse, nous semble alors ouvrir une perspective intéressante à questionner : cela permettrait de mettre en évidence le rôle d'acteurs des Roms et, plus généralement, la portée politique des pratiques urbaines de ces populations et leur quête de citadinité.

À Turin comme à Marseille, nos deux terrains d'étude, on recense plusieurs lieux d'habitat précaire où des migrants roms se sont installés au fil du temps ${ }^{10}$. Ces installations précaires mobilisent des espaces dits marginaux, des interstices, des replis, des espaces délaissés et négligés (terrains vagues, bâtiments abandonnés, friches industrielles, usines désaffectées) en raison du peu d'intérêt qu'ils représentent pour les autorités planificatrices et régulatrices et les investisseurs immobiliers et fonciers ${ }^{11}$. Que ce soit à travers l'installation de caravanes, de cabanes, par l'occupation de bâtiments ou tout simplement du trottoir, ces espaces permettent néanmoins à ces personnes de s'implanter dans la ville ${ }^{12}$.

8. Legros O., Olivera M., «La gouvernance métropolitaine à l'épreuve de la mobilité contrainte des "Roms migrants" en région parisienne ", EspacesTemps.net, Travaux, 21 mars 2014 (www.espacestemps.net/articles/lmobilite-contrainte-des-roms-migrants-en-regionparisienne/, consulté le 26/06/2015).

9. Hernando De Soto parle notamment des «slums » des pays du Sud comme des lieux habités par des entrepreneurs héroïques, $c f$. De Soto H., The Mystery of Capital: Why Capitalism Triumphs in the West and Fails Everywhere Else, New York, Basic Books, 2000.

10. Il s'agit pour la plupart de migrants roms provenant des pays d'Europe de l'Est (notamment de Roumanie), arrivés dans les villes occidentales à partir de la seconde moitié des années 2000, compte tenu des spécificités qui caractérisent chaque contexte. Par exemple, l’arrivée de familles roms de Roumanie à Turin est déjà signalée au cours des années 1990. Cf. Revelli M., Fuoriluogo, Turin, Bollati Boringheri, 1999.

11. Roulleau-Berger L., La Ville-Intervalle : jeunes entre centre et banlieue, Paris, MéridiensKlincksieck, 1993 [1991]. Baby-Collin V., Bénit C., Bouillon F., Grésillon B., Rinaudo C., «Détourner », in Dorier-Apprill E., Gerais-Lambony P. (dir.), Vies citadines, Paris, Belin, 2007, pp. 81-108.

12. Il faut préciser les limites de cette généralisation qui ne tient pas compte des Roms qui n’habitent pas dans un habitat précaire, ni des trajectoires résidentielles des individus et des familles, se concentrant exclusivement sur leur situation actuelle. 
Deux établissements illégaux en particulier ont fait l'objet de cette étude : le campement Lungo Stura à Turin et le bidonville de la Capelette à Marseille ${ }^{13}$. Elle a été menée au moyen d'une enquête ethnographique afin d'analyser la marginalité "par le bas », en suivant le déroulement de la vie quotidienne des individus. Dans le cas de Turin, qui a été traité plus en profondeur, l'ethnographie s'est déroulée en deux moments différents, de juillet à septembre 2013 et de mars à octobre 2014. Quant au cas de Marseille, qui fait au moment de la rédaction de cet article (juin 2015) l'objet spécifique de notre recherche, sont ici repris les résultats des enquêtes préalables conduites en juin 2013 et en mai 2014. Dans les deux cas, le regard a porté sur les manières dont les migrants roms utilisent, traversent et s'installent dans les espaces de la ville. À partir de leurs lieux de vie, nous avons rencontré et observé les Roms dans leurs déplacements quotidiens en bus, en vélo et à pied ; aux marchés aux puces; dans les salles d'attente des bains-douches municipaux ; aux croisements des rues, devant les magasins, aux coins des trottoirs. L'observation a été complétée par des entretiens non directifs afin de saisir le sens que les personnes donnent à leurs pratiques et les motivations qui en déterminent le développement. Cette approche permet d'étudier le sens de la résistance en pratique, en interrogeant les modalités selon lesquelles elle s'articule et la manière dont elle est vécue eu égard aux processus de marginalisation portés par les politiques publiques.

\section{Le refus de la marginalisation. Résister discrètement à la précarité}

S'installer et s'implanter. "Et pourquoi devrais-je partir? Ici, je suis chez moi ${ }^{14}$ "

Les cas turinois et marseillais présentent des caractéristiques très différentes quant aux conditions d'habitat des migrants roms, déterminées par les spécificités de chaque contexte socio-spatial et économique, auxquelles s'ajoutent les effets des politiques et des actions publiques en direction de ces migrants. À Marseille, la succession des expulsions des squats et bidonvilles demeure l'aspect le plus évident, générant au cours du temps des conditions extrêmes de précarité, une mobilité quasi permanente et forcée des familles d'un site à l'autre et donc leur dispersion dans la ville.

À l'inverse, dans le cas de Turin, c'est la concentration spatiale qui caractérise de manière flagrante la configuration de l'habitat précaire des Roms. Elle résulte d'une volonté publique bien réelle, quoique non dite. En effet, les autorités administratives ont, d'un côté, piloté les expulsions des sites illégaux et, de l'autre, assuré la gestion des campi nomadi autorisés ${ }^{15}$ ainsi que l'offre

13. La différenciation entre «campement » et «bidonville » est un choix explicite qui renvoie aux mots les plus souvent utilisés dans les deux pays pour désigner les établissements roms illégaux : «campo » en italien et « bidonville» en français.

14. Dorin, notes de terrain, Marseille, juin 2013. 
de services publics (santé, scolarisation des enfants, accompagnement aux démarches administratives), de sorte que la plupart des établissements où habitent les Roms sont aujourd'hui concentrés dans une partie spécifique de la ville. Situé dans la périphérie nord-est, ce territoire est traversé par la rivière Stura dont les berges sont devenues au cours des dernières décennies une zone d'activités reléguées : on y retrouve la décharge publique, la fourrière municipale et d'autres activités non autorisées, telles le dépôt sauvage d'ordures et des potagers informels. Qu'ils aient volontairement fait le choix de marginaliser des activités qu'ils souhaitaient éloigner du centre-ville ou qu'ils aient tacitement décidé d'ignorer le caractère illégal de certaines activités, les pouvoirs publics ont donc toléré, voire permis le développement de l'habitat précaire et son ancrage dans l'espace et dans le temps.

Selon les prévisions du plan local d'urbanisme, le territoire où est installé le campement Lungo Stura est destiné à devenir un parc public, aucune opération immobilière n'étant possible car il s'agit d'un terrain soumis à un risque d'inondation. Jusque-là, aucune transformation n'a été initiée. Dans l'attente, d'autres dynamiques ont suivi leur propre cours : c'est dans ces espaces de non-intervention que les Roms ont trouvé des possibilités pour s'installer et demeurer ${ }^{16}$.

Le campement Lungo Stura existe depuis 2005-2006. En 2013, le site abritait environ huit cents personnes ${ }^{17}$. Les matérialités qui le structurent nous renseignent sur un ancrage et une permanence qui sont tout d'abord physiques. Les habitations, réalisées avec des matériaux récupérés, ont acquis au cours du temps une certaine solidité. En témoignent leur taille, le ciment et le carrelage utilisés pour aménager le sol, la décoration des espaces extérieurs avec des pots de fleurs aux fenêtres, et intérieures avec des tapis, des portraits religieux, des napperons sous la télé. Quelques cabanes sont destinées à des utilisations non résidentielles, notamment des espaces de vente de snacks et boissons ${ }^{18}$, un espace pour les activités des associations et quatre lieux de culte (un orthodoxe, deux évangéliques et un baptiste). Une des premières familles à construire sa cabane dans ce campement était celle de Madalina. Comme elle nous a expliqué, elle a quitté la Roumanie en 2005 et est arrivée ici il y a dix ans avec son mari et ses trois enfants. Dès lors, « nous sommes toujours là, dans la même cabane, dans le même endroit... les enfants sont allés à l'école ici... ici, c'est chez nous ${ }^{19}$ ».

15. Les campi nomadi, littéralement « campements pour nomades », sont des dispositifs d'accueil mis en œuvre par les administrations publiques italiennes à partir des années 1980, qui formalisent la mise en marge spatiale des Roms, $c f$. Sigona N., "Locating "The Gipsy Problem". The Roma in Italy: Stereotyping, Labelling and "Nomad Camps" ", Journal of Ethnic and Migration Studies, vol. 31, nº 4, 2011, pp. 741-756.

16. Legros O. et Olivera M., op. cit.

17. Comune di Torino, Capitolato speciale d'appalto per la gestione di iniziative a favore della popolazione rom, Turin, 2013.

18. Sorte de bars, ces cabanes sont périodiquement fermées par les autorités, car sans autorisation à la vente. 
Situé dans le 10e arrondissement marseillais, le bidonville de la Capelette comptait, au moment de la dernière expulsion en octobre 2013, environ quatre cents personnes, pour la plupart des Roms roumains, arrivées sur le terrain à partir d'octobre 2012. Il se trouvait sur une friche industrielle localisée à côté d'une déchetterie et séparée de celle-ci par le chemin de fer qui sert aujourd'hui à l'évacuation des détritus de la ville. Ce même terrain était déjà squatté par une quinzaine de familles roms en 2011. Entre 2009 et 2013, trois squats et quatre bidonvilles se sont succédés à la Capelette, au gré des expulsions et des réimplantations multiples qui transforment discrètement les espaces délaissés du quartier. Ce faisant, ces dernières s'inscrivent dans d'autres temporalités des transformations urbaines. Ancien quartier ouvrier, la Capelette connaît aujourd'hui d'importants processus de reconversion et de renouvellement urbain. La construction de quatre mille nouveaux appartements, dans le cadre d'un projet d'une zone d'aménagement concerté et d'aménagement d'espaces de loisir (le Palais omnisport Marseille Grand-Est a ouvert en 2009), permettront au quartier de ne plus faire office de quartierdortoir et de se revitaliser. La présence d'espaces «vides ", dépossédés de leur fonction productive et en attente d'une nouvelle affectation, semble bien être la ressource principale à la fois pour les transformations urbaines et les acteurs qui y prennent part, et pour les migrants roms qui peuvent s'y installer. L'idée d'espace-réserve avancée par Núria Benach et Rosa Tello nous indique une piste intéressante à ce propos : ces espaces en attente, en réserve de projets d'urbanisme, seraient délibérément laissés à l'abandon pour pouvoir ensuite justifier leur mise aux normes à travers des transformations 20 .

Les expulsions qui se succèdent à la Capelette sous prétexte de transformations urbaines obligent les Roms à se déplacer d'un terrain à l'autre, d'une friche à l'autre. Ainsi, de squats en squats, de bidonvilles en bidonvilles, et malgré les conditions de précarité auxquelles ils sont exposés, ils ne quittent pas le quartier. On estime en effet qu'entre 2009 et 2013, certaines familles se sont déplacées au moins cinq ou six fois. En se cantonnant à ce périmètre de cinq cents mètres carrés, ils témoignent d'un ancrage assez fort au quartier, jusqu'à ce qu'il n'y ait plus d'espaces disponibles. Une des premières familles à arriver dans le quartier est celle de Dorin, à la fin 2009. Avec trois autres familles, ils s'étaient installés dans un bâtiment préfabriqué appelé « La Pauline ». Le squat est expulsé en mai 2011, ils commencent alors cette errance forcée à travers les espaces vides du quartier. Lors de notre enquête en juin 2013, l'expulsion des familles de la Capelette était imminente. Des militants avaient proposé à la famille de Dorin de partir ailleurs, vers Cassis où il y avait la possibilité de s'installer sur des terrains vagues, loin du regard des autorités, mais Dorin refusait de quitter la ville : « là-bas, on connait personne, nous, on

19. Notes de terrain, Turin, septembre 2014.

20. Benach N., Tello R., "Des espaces-réserve versus des espaces de résistance ? ", in Semmoud N., Florin B., Legros O., Troin F. (dir.), Marges urbaines et néolibéralisme en Méditerranée, Tours, Presses universitaires François Rabelais, 2014, pp. 43-57. 
parle pas bien le français... ici on a des relations, la famille, le groupe... on se sent protégés... on va pas les laisser 21 ».

En dépit des différences constatées, il apparaît dans les deux cas étudiés que les espaces de vie sont construits de manière individuelle et incrémentale, selon un processus progressif d'assemblage de différentes matérialités récupérées dans la ville. Ainsi, cette dernière est «soit exploitée soit recyclée [...] dans un contexte de distribution inégale de ressources 22 ». En tant que formes incrémentielles d'habitations urbaines, ces lieux occupés peuvent être lus comme une manière d'adaptation au caractère ordinaire de la crise ${ }^{23}$. La notion de « précarité » souvent utilisée pour décrire, voire dénoncer les conditions d'habitat des Roms ${ }^{24}$ se réfère à leur situation générale de détresse et de mal-logement. Ce terme exprime par ailleurs l'incertitude et l'insécurité à la fois socio-économique et affective, psychologique et perceptive. En d'autres termes, la précarité renvoie à une forme d'insécurité qui recouvre toutes les dimensions de l'existence. C'est précisément cette vision qui incite à analyser les réactions et les résistances des populations précaires à ces conditions de vulnérabilité 25 .

\section{Pallier le manque d'eau. "Nous? On les mange pas les poissons qu'on a pris 26 »}

À Turin, l'eau de la rivière offre plusieurs ressources aux personnes qui habitent sur les berges de la Stura : les femmes y font la vaisselle et la lessive, les enfants l'utilisent comme piscine pendant la saison chaude. Comme nous le raconte Sergiu, « il y a plein de poissons, tu vois, moi je mets ça dans l'eau [il nous montre un seau auquel il a fait des trous, il sert comme filet de pêche avec une corde fixée au bord pour le trainer], puis quand les poissons y vont dedans je tire... et voilà je le sors de l'eau avec les poissons dedans... mais on les mange pas, je les remets tout de suite dans l'eau, c'est juste pour jouer 27 ». La rivière permet aussi de se laver. Ainsi, un jeudi de septembre, Mariana nous invite à prendre un café chez elle, c'est la première fois que nous rencontrons son mari. Il entre dans la cabane chercher son paquet de cigarettes. Le temps d'en fumer une, Mariana lui prépare un petit sac avec une serviette, du linge,

21. Notes de terrain, Marseille, juin 2013.

22. "The city is both mined and recycled [...] in context of deeply unequal resources and precarious lives ». McFarlane C., «Assemblage and critical urbanism », City, vol. 15, n², 2011, p. 216 (notre traduction).

23. Berlant L., Cruel Optimism, Durham, Duke University Press, 2011.

24. Legros O., op. cit. ; Legros O., Rossetto J., « La “question rom” en Europe aujourd'hui : regards croisés et mises en perspectives ", Études Tsiganes, n 46, 2011, pp. 4-25. Vitale T.,

"Les politiques locales face aux Roms : entre réification, effets de visibilité et reconnaissance », Métropolitiques, 4 février 2015 (www.metropolitiques.eu/Les-politiques-localesface-aux.html, consulté le 26/06/2015).

25. Simone A. M., City Life from Jakarta to Dakar, Londres/New York, Routledge, 2010.

26. Sergiu, notes de terrain, Turin, septembre 2014.

27. Notes de terrain, Turin, septembre 2014. 
du shampooing, puis il part, sans rien dire, elle nous explique à voix basse qu'il va prendre une douche à la rivière. La rivière fait également office de toilettes. La végétation qui entoure les berges permet de soustraire ces pratiques aux regards extérieurs. Le fait d'être à l'abri des regards, de ne pas attirer l'attention explique pour partie l'usage de l'adjectif « discret 28 ».

À Turin, les fontaines publiques sont la principale source d'eau potable pour les Roms. Les multiples petites fontaines vertes dont l'eau jaillit d'une tête en forme de taureau, sont l'un des symboles de la ville. Ainsi, toute personne a la possibilité d'accéder gratuitement à l'eau potable, y compris les Roms et les autres groupes ou individus à l'habitat précaire (sans-abri, squatteurs) qui utilisent ces mêmes fontaines pour se laver, faire la lessive et la vaisselle ${ }^{29}$. La distance moyenne qu'une personne habitant Lungo Stura doit parcourir pour accéder à une fontaine est d'environ cinq cent mètres. Au moins une fois par jour, les habitants de Lungo Stura vont chercher l'eau à l'aide de gros bidons en plastique, qu'ils transportent dans de vieilles poussettes récupérées ou accrochés sur le porte-bagage des vélos.

Les Roms ont également pour habitude d'utiliser les quatre bains-douches municipaux de la ville. Comme nous avons pu l'observer, ils s'y rendent plusieurs fois par semaine et pas uniquement pour s'y doucher. Il s'agit plutôt d'un temps de repos passé en famille. Ils y restent en effet deux ou trois heures à chaque fois, le temps de se laver mais aussi de s'habiller, de se sécher, de se brosser les cheveux pour les femmes, et de prendre un chocolat chaud au distributeur automatique pour les enfants qui les accompagnent.

À Marseille, l'eau potable est bien moins accessible. La pénurie de fontaines et l'absence de bains-douches publics représentent une véritable entrave à l'hygiène personnelle, ce qui est d'ailleurs dénoncé par les associations locales. Face à ces difficultés, les bouches d'incendie permettent souvent aux Roms un accès illégal mais régulier à l'eau, ce qui implique plus qu'ailleurs des pratiques de contournement et des savoir-faire techniques. Ces compétences que l'on peut juger précaires car acquises en situation de contrainte $30-s^{\prime}$ expriment dans le repérage des bornes, dans leur détournement et dans les stratégies d'invisibilisation de cette pratique compte tenu de son caractère illégal : aller chercher l'eau très tôt le matin ou assez tard le soir; utiliser des bouteilles d'eau vides plutôt que des gros bidons pour ne pas attirer l'attention.

28. «Qui n'attire pas l'attention, qui ne se fait guère remarquer », cf. Le Petit Robert de la langue française, 2013.

29. Pour l'usage des fontaines publiques d'une manière subversive, voir : Kowalewski M., "Producing the Space, Contesting the City: Urban Wild Swimming ", in Gulçin Lelandais (dir.), Understanding the City: Henri Lefebure and Urban Studies, Newcastle, CSP, 2014, pp. 171-187.

30. Bouillon $\mathrm{F}$., «Les compétences précaires en question : réflexions à partir des squats marseillais », in Isabelle Berry-Chikhaoui et al. (dir.), Villes internationales, entre tensions et réactions des habitants, Paris, La Découverte, 2007, pp. 189-206. 
Prenant appui sur des travaux récents de chercheurs anglo-saxons ${ }^{31}$, il nous semble important de souligner ici la relation entre d'un côté l'approvisionnement en eau, l'assainissement et l'hygiène personnelle et, de l'autre, l'expérience corporelle (embodied experience) vécue du fait du manque d'équipements. Les rapports entre corporalités et infrastructures sont en l'occurrence compris sous le prisme de la relation entre les réalités matérielles de la vie quotidienne et les structures politiques et socio-économiques. Porter son attention à la matérialité des corps permet de mettre en évidence les aspects actifs, transformatifs et pratiques de la corporalité comme éléments des relations de pouvoir ${ }^{32}$. Se soigner et se laver malgré le manque d'eau, y compris prendre une douche à la fontaine publique, révèle l'importance de la corporalité dans l'expérience de la marginalité telle qu'elle est à la fois véhiculée et contestée par les pratiques quotidiennes des populations des marges. Ainsi plutôt que de parler de marginalité subie - et tout en reconnaissant les conditions d'exclusion et les ségrégations qui affectent les Roms et auxquelles ils doivent se confronter quotidiennement, nous préférons parler de marginalité agie par les pratiques spatiales et vécue à travers le corps.

\section{Récupérer les déchets. "Faut toujours garder ses mains propres 33 "}

«Faire les poubelles» est l'une des activités les plus répandues chez les Roms, à Turin, à Marseille comme ailleurs. Elle comprend la récupération des métaux, qui consiste selon leurs termes à « faire la ferraille ». Ce travail de récupération s'effectue selon plusieurs modalités : il peut se faire par l'évacuation de locaux, le débarras de caves, le don. Dans tous les cas, la fouille des poubelles reste la pratique la plus courante. Les éléments récupérés sont très variés : objets en métal (articles ménagers, ustensiles de cuisine, pièces de rechange pour les téléphones portables), vêtements et chaussures, aliments, livres ou bandes dessinées.

La dimension spatiale de la récupération des déchets, y compris le tri et la vente, est l'un des aspects les plus importants de cette activité qui se pratique dans divers lieux urbains. La majorité des Roms que nous avons rencontrés à Turin cherche sa matière première dans les bennes à ordures de certains quartiers, généralement les plus aisés, car c'est là que se trouvent les rebuts les plus intéressants. La fouille des poubelles, qui se fait à pied ou à vélo, implique une

31. McFarlane C., "Metabolic inequalities in Mumbai. Beyond telescopic urbanism ", City, vol. 17, n²4, 2013, pp. 498-503. Desai R., McFarlane C., Graham S., «The Politics of Open Defecation: Informality, Body and Infrastructure in Mumbai », Antipode, vol. 47, n¹, 2014, pp. 98-120. McFarlane C., Vasudevan A., « Informal infrastructures », in Adey P., Bissell D., Hannam K., Merriman P., Sheller M. (eds), Handbook of Mobilities, Abingdon/New York, Routledge, 2014, pp. 256-264.

32. Shinko R. E., Theorizing Embodied Resistance Practices in International Relations (in progress) (https://www.sussex.ac.uk/webteam/gateway/file.php?name=theorizing-embodiedresistance-practices-in-international-relationsedited.pdf\&site=12, consulté le 26/06/2015).

33. Dario, notes de terrain, Turin, mai 2014. 
connaissance approfondie de la ville, de ses espaces et de ses temporalités, nécessaire pour une bonne organisation du travail de récupération. Les zones de la ville où une collecte sélective à domicile est organisée sont à éviter, car il n'y a plus de poubelles dans la rue. Dans tous les cas, il faut connaître l'horaire $\mathrm{du}$ passage des camions de la collecte officielle, pour arriver avant que les poubelles ne soient vidées. Si ce travail permet aux Roms de traverser et d'utiliser plusieurs espaces de la ville - rues, places, marchés - à des moments différents de la journée, ces pratiques et ces parcours les placent toutefois dans une «ville à l'envers ${ }^{34}$ ».

Ainsi travaillent-ils également pendant la nuit, pour collecter les déchets avant que les camions-bennes ne passent. C'est ce que nous explique Veronica quand elle nous raconte sa journée : la récupération des déchets se fait le matin entre $5 \mathrm{~h}$ et $8 \mathrm{~h}$ environ, et le soir entre $18 \mathrm{~h}$ et $22 \mathrm{~h}$. Entre-temps, elle fait le ménage, prépare à manger, s'occupe des enfants.

C'est à l'heure de fermeture des marchés, des magasins, des centres commerciaux, qu'on a la possibilité de récupérer ce que jettent les commerçants, que ce soit des déchets alimentaires ou des vêtements. Par exemple, au marché alimentaire de Porta Palazzo, le plus grand de Turin, nous avons pu observer des femmes roms qui attendaient le boucher à la fin de la journée, devant la porte de service. Ce dernier sortait avec un grand récipient en plastique contenant les restes de viande. Alors que les camions de collecte des déchets alimentaires étaient déjà au travail, les femmes roms sélectionnaient la viande, puis jetaient le reste à la poubelle. Cela se répétait presque tous les jours. Mariana nous racontait aussi qu'elle allait récupérer les cartons d'emballage dans les centres commerciaux pour les revendre ensuite aux industries qui produisent du papier recyclé. Puisqu'ils sont habituellement stockés dans des espaces situés à l'arrière du bâtiment principal, elle y accédait par là le soir ou la nuit pour ne pas être vue.

La perception du stigmate qui frappe ce travail de récupération se manifeste au travers de plusieurs éléments. En effet, lors de notre première rencontre avec Nicolina, elle nous explique que sa mère est partie faire les courses, alors que cette dernière revient peu après à Lungo Stura chargée d'un panier rempli d'objets récupérés dans les poubelles. Elle nous raconte que « Nicolina, elle est venue une seule fois avec moi faire les poubelles, elle avait peur de rencontrer ses copains, elle avait honte... elle n'est plus jamais venue avec moi... et moi, tu sais, quand j'ai terminé le travail que je prends le bus, dans le bus tout le monde s'éloigne de moi, je les sens les gens parler de moi 35 ». Ou

34. Cette expression est reprise d'une recherche menée par le «Bureau de l'envers », un groupe de jeunes architectes marseillais qui reconstruisent les déplacements d'une famille rom en ville (http://cargocollective.com/lebureaudelenvers/, consulté le 29 juin 2015).

35. Veronica, notes de terrain, Turin, avril 2014. 
encore, quand nous croisons Dario dans la rue, en train de fouiller les bennes, il prend soin de retirer ses gants avant de nous tendre la main pour nous saluer. Florian qui, lui, travaille sans gants, nous tend son coude.

À l'activité de récupération succède le tri : après avoir sélectionné les objets en bon état, séparé les différents types de métaux et identifié les objets à réparer, chaque produit suit son propre parcours dans les filières de l'économie formelle ou informelle et parfois illégale. De nombreux produits sont vendus sur les principaux marchés aux puces des deux villes (les Arnavaux à Marseille et le Balôn à Turin). Redonner une vie aux déchets grâce à des compétences pratiques qui «sauvent » les objets en les réintroduisant dans les circuits économiques permet d'organiser un « contre-stigmate moral 36 » vis-àvis des personnes qui se les procurent et donc qui, d'une certaine façon, rachètent leurs propres déchets. Ainsi, quand nous nous rendons chez Somna pour l'accompagner à l'église un dimanche après-midi, elle considère que nos chaussures ne sont "pas jolies ni propres » pour ce moment de fête ${ }^{37}$. Nous nous voyons donc offrir une autre paire de chaussures à talons hauts décorées d'une rosette, qu'elle a bien nettoyée après l'avoir récupérée.

Le stigmate associé aux Roms qui font de la récupération des déchets leur activité principale s'explique non seulement par la visibilité des personnes qui fouillent les bennes dans la rue, mais aussi du fait des ordures qui entourent leurs lieux de vie. Ils sont ainsi suspectés de miner l’ordre « post-hygiéniste 38 » fondé sur la dimension sociale et morale de la propreté urbaine : une ville propre est une ville sûre, tranquille, structurée suivant des normes qui ne tolèrent pas l'amoncellement de déchets irréguliers ${ }^{39}$. Cela renvoie à la question de la visibilité/invisibilité de certaines pratiques et des Roms dans la ville. Comme le remarquent Olivier Legros et Tommaso Vitale ${ }^{40}$, bien que la présence de ces groupes soit relativement modeste (entre 15000 et 20000 en France et environ 60000 en Italie, pour les Roms roumains), ils ont une forte visibilité dans l'espace urbain, ce qui fonde la construction du problème public, c'est-à-dire de la « question rom » et des politiques répressives dont ils font l'objet.

36. Le Lay S., " Enseignements empiriques et éthiques d'une biffe sociologique parmi les éboueurs parisiens », Actes de la recherche en sciences sociales, vol. 5, n²05, 2014, p. 123.

37. Notes de terrain, Marseille, mai 2015.

38. Kokoreff M., «La propreté du métropolitain. Vers un ordre post-hygiéniste ? », Les Annales de la recherche urbaine, $\mathrm{n}^{\circ} 53,1991$, pp. 92-102.

39. Milliot V., "La ville informelle ou le travail des marges ", in Milliot, V., Tastevin, Y. P. (dir.), Les archipels de la Goutte d'Or. Analyse anthropologique d'une "métropolisation par le bas", Programme de recherche "Cultures et territoires en Ile-de-France », Rapport final, 2010, pp. 7-59.

40. Legros O., Vitale T., « Les migrants roms dans les villes françaises et italiennes : mobilités, régulations et marginalités », Géocarrefour, vol. 86, n¹, 2011, pp. 3-14. 
Au marché aux puces, les Roms remettent les objets récupérés dans les circuits économiques des gadjés (les étrangers, les non-Roms). Dans ces espaces ils deviennent des vendeurs comme les autres, italiens, français, maghrébins, une condition d'anonymat qui les éloigne de l'image-stigmate des personnes qui fouillent les poubelles. L'anonymat fonctionne ici comme tactique ${ }^{41}$ qui leur permet non seulement d'ouvrir une relation avec les personnes qui viennent acheter, mais aussi d'organiser le contre-stigmate dont on faisait mention précédemment.

De plus, la pratique des espaces variés de la ville qui résulte de l'activité de récupération peut être considérée en terme d' ' empiètement tranquille de l'ordinaire ", une expression qu'Asef Bayat utilise pour décrire l'avancement silencieux et prolongé des personnes ordinaires dans les espaces de pouvoir, de la propriété, dans les espaces publics, afin d'améliorer leur condition de vie $^{42}$.

Parcourir la ville. "Pas grave si je me perds 43 "

Que ce soit pour la récupération des déchets, pour prendre une douche aux bains municipaux ou pour vendre des affaires aux marchés aux puces, les migrants roms parcourent la ville entière. À Turin, dans la plupart des cas, les déplacements se font en vélo ou en transports en commun. Le vélo est notamment utilisé pour l'activité de récupération des déchets. Là encore, on se retrouve face à une inversion des manières de parcourir la ville, car la plupart des récupérateurs qui utilisent le vélo n’hésitent pas à prendre la route en contresens ou à rouler sur le trottoir quand cela leur permet d'accéder aux poubelles plus aisément.

De plus, les Roms que nous avons rencontrés ont montré une connaissance profonde des réseaux de transport en commun qui deviennent un point de référence et de repère fondamental pour leur pratique de la ville. Lorsqu'ils doivent expliquer ou décrire un chemin spécifique, ils utilisent les numéros de bus et des arrêts plutôt que les noms de rues et de places. Si, pour certains d'entre eux, cela peut s'expliquer par leur analphabétisme, les jeunes rencontrés à Turin étaient scolarisés depuis dix ans, ils savaient lire, mais ils partageaient les mêmes points de repère que les autres. Ionut a 12 ans, il fait partie d'une équipe de football avec d'autres jeunes Roms qui habitent dans un autre campement non loin de Lungo Stura. Quand il parle d'eux, il les appelle « ceux du 51 », du numéro du bus qui s'arrête devant l'autre campement.

41. Secor A., " "There Is an Istanbul That Belongs to Me": Citizenship, Space, and Identity in the City ", Annals of the Association of American Geographers, vol. 94, n², 2004, pp. 352368.

42. "A quiet encroachment of the ordinary " (Bayat A., Life as Politics. How Ordinary People Change the Middle East, Amsterdam, Amsterdam University Press, 2010, p. 43 - notre traduction).

43. Mariana, notes de terrain, Turin, avril 2014. 
Parfois, il leur arrive de se perdre, notamment quand ils fouillent les poubelles, car leur attention est entièrement focalisée sur la récupération. C'est alors que les réseaux de bus deviennent un filet de protection : « je fais les poubelles, je marche, je marche, puis à un moment donné je ne sais plus où je suis... mais t'inquiète, je sais pas dire comment je fais, mais le soir je rentre à la maison, toujours... je rejoins l'arrêt de bus le plus proche, n'importe lequel, je demande au chauffeur, si c'est bien pour aller vers le $51 \ldots$ puis je monte... et je rentre ${ }^{44}$ ». Marius, un homme de 60 ans rencontré devant l'entrée d'un supermarché où il faisait la manche, parlait peu l'italien, et la seule information claire que nous avons reçue de sa part c'était « le tram... 18, 27, 49... le tram... 18, 27, 4945 ». Selon Gilles Deleuze et Felix Guattari, la ritournelle fonctionne comme un cercle qui se forme autour du sujet, à la fois pour s'éloigner du chaos, pour se maintenir «chez soi » et enfin pour s'ouvrir au monde : " on n'ouvre pas le cercle du côté où se pressent les anciennes forces du chaos, mais dans une autre région, créée par le cercle lui-même. Comme si le cercle tendait lui-même à s'ouvrir sur un futur, en fonction des forces en œuvre qu'il abrite. Et cette fois, c'est pour rejoindre des forces de l'avenir, des forces cosmiques 46 ». La ritournelle fonctionne ici comme une stratégie discrète de résistance envers une déterritorialisation trop puissante et déstabilisante : il en est de même pour le fait d'apprendre à se repérer dans la ville et à se la représenter à travers les réseaux de transport en commun, utilisés comme des points de repère alternatifs.

\section{Faire la manche. "Ce trottoir, ça fait huit ans que je viens ici 47 "}

Plusieurs Roms rencontrés faisaient la manche. S'il n'est pas question ici d'analyser plus en avant ce que la mendicité incarne et les différentes manières dont elle est pratiquée ${ }^{48}$, il est possible de mettre en exergue ce qui a trait à son articulation spatiale et les interactions sociales qui en résultent. La mendicité peut être une pratique fixe ou en mouvement. Dans le cas des personnes que nous avons rencontrées, la mendicité se pratique soit de manière statique et ponctuelle, notamment en restant assis à l'entrée des hôpitaux, magasins, églises, supermarchés, centres commerciaux, soit en mouvement, ce qui est le cas des Roms qui font la manche aux feux de circulation. Dans ce dernier cas, le mouvement se routinise, trouve un rythme propre ${ }^{49}$ qui se répète tout au long de la journée, en avant et en arrière suivant le flux des voitures et l'alternance des feux.

44. Mariana, notes de terrain, Turin, avril 2014.

45. Notes de terrain, Turin, août 2014.

46. Deleuze G., Guattari F., Mille Plateaux. Capitalisme et schizophrénie 2, Paris, Les Éditions de Minuit, 2013 [1980], pp. 382-383.

47. Stefania, notes de terrain, Turin, mai 2014.

48. Dean H. (eds), Begging Questions. Street-Level Economic Activity and Social Policy Failure, Bristol, The Policy Press, 1999.

49. Desai R., McFarlane C., Graham S., op. cit. 
Plusieurs aspects contribuent au choix du lieu mais l'élément fondamental s'avère être l'interaction avec les gens, à la fois en termes de quantité (combien de personnes/voitures sont censées passer par là) et de qualité (type d'interaction avec les riverains, les commerçants du quartier). La permanence quotidienne du mendiant sur le même coin de trottoir, la même marche ou le même croisement de rues habitue les passants fréquents à sa présence, ce qui permet de développer plus de liens de confiance avec eux. C'est notamment dans ces relations mendiant/individu qui font le don que James Scott repère la mise en place de résistances dites stratégiques ${ }^{50}$, dans le sens où les subalternes usent de la rhétorique des riches et des puissants (leur idée de devoir ou de charité) pour obtenir des dons ${ }^{51}$.

Les pratiques routinières et rythmées des espaces de la ville révèlent les manières dont les Roms se les approprient, discrètement mais assez efficacement. Stefania fait la manche sur la place Emanuele Filiberto à Turin : «j'y viens depuis huit ans, oui je suis arrivée à Turin en 2004, puis le temps de m'installer... et dès lors, c'est toujours ici que je viens, sur le même coin de trottoir 52 ». Revenir sur le même lieu tous les jours pendant des années, nous renseigne par rapport à ces appropriations silencieuses. Des considérations identiques pourraient être faites au sujet de Rodriga, qui fait la manche à un feu de circulation. Pendant l'été, quand elle part en Roumanie, c'est son fils qui prend sa place, en assurant ainsi une continuité spatio-temporelle qui prévient l'occupation par d'autres personnes. À ce propos, il est également important de souligner que les lieux occupés par les mendiants sont très souvent marqués par leur présence même quand ils ne sont pas là. En effet ils laissent habituellement un pull, un bout de carton, une bouteille d'eau ou d'autres types d'objets qu'ils utilisent pour travailler et qui deviennent des signes de permanence et d'appropriation.

Si la pratique de la mendicité est souvent lue en tant que stratégie de survie (plutôt que de résistance), Asef Bayat souligne qu'accorder une importance excessive à cette interprétation peut contribuer à maintenir l'image des pauvres comme victimes, déniant leur capacité d'action. Ce que nous nous attachons à montrer, ce sont, au contraire, les formes de résistances liées à cette pratique si répandue chez les Roms que nous avons rencontrés. En effet, la pratique de l'espace qui résulte du fait de mendier tous les jours sur le même coin du trottoir s'avère être une stratégie de revendication, une affirmation de leur pouvoir d'action à travers l'appropriation de l'espace ${ }^{53}$. Ce faisant, ils mettent en défi d'une part la tentative d'invisibilisation véhiculée par les poli-

50. Il fait notamment référence aux pratiques de mendicité développées par les paysans en Malaisie, interprétées comme des formes de résistance stratégique à l'agriculture capitaliste.

51. Scott J. C., Weapons of the Weak: Everyday Forms of Peasant Resistance, New Haven, CT, Yale University Press, 1985.

52. Notes de terrain, Turin, mai 2014.

53. De Certeau M., L'Invention du quotidien. Arts de faire, Paris, Gallimard, 1990 [1980]. 
tiques publiques qui les relèguent dans des espaces marginaux. D'autre part, ils revendiquent une appropriation de l'espace public qui leur est souvent niée par les normes qui en règlent l'utilisation, comme c'est par exemple le cas des arrêtés contre la mendicité que la Municipalité de Marseille a mis en œuvre à plusieurs reprises dans le centre-ville.

\section{Mise en perspective. Une quête de citadinité ?}

La plupart des études consacrées à la « question rom » en Italie et en France mettent l'accent sur les processus de marginalisation dont ces migrants sont victimes et sur les conditions de vulnérabilité et de précarité qui en résultent. Ces «politiques d'usure » produiraient, selon certains auteurs, des effets d'auto-expulsion des familles qui finiraient par s'éloigner des villes où elles se sont installées, voire rentrer dans leurs pays d'origine ${ }^{54}$. Si l'on ne peut pas nier les effets majeurs des politiques « roms » sur les trajectoires des individus et des groupes, notamment en termes d'errance forcée à la suite des expulsions, la permanence de ces sites et de ces personnes dans la ville mérite d'être prise en compte différemment. L'ancrage territorial est en effet l'élément le plus saillant de notre analyse des pratiques de résistance des migrants roms.

Ces pratiques ne sauraient se comprendre comme une opposition déclarée entre les puissants et les faibles, ni comme des luttes menées dans les espaces construits par les relations structurantes ${ }^{55}$. Au contraire, c'est précisément grâce aux discontinuités des espaces de pouvoir, aux asymétries territoriales ${ }^{56}$, qu'elles peuvent se développer discrètement et résister à la précarité et à la marginalité, en revendiquant silencieusement le désir de trouver une place dans la ville. Ces espaces de résistance sont multiples, dynamiques et seulement en partie contrôlés par les pratiques de domination. C'est ce que James Scott appelle « l'infrapolitique des dominés », où se développe « une grande variété de formes discrètes de résistances qui n'osent pas dire leur nom 57 », qui ne s'opposent pas aux pouvoirs de manière ouverte, qui restent en partie invisibles, mais dont la portée subversive ne doit pas être remise en question. À travers ces entre-deux spatiaux, sociaux, politiques et économiques, elles « tracent les ruses d'intérêts autres et de désirs qui ne sont ni déterminés ni captés par les systèmes où elles se développent 58 ». Ce faisant, ces pratiques témoignent des capacités des Roms à résister aux contraintes et à la marginalisation venue "d'en haut ». Elles doivent ainsi être reconnues en tant que revendication en acte de citadinité ${ }^{59}$, porteuses d'un processus constant d'af-

54. Fassin É., Fouteau C., Guichard S., Windels A., Roms E riverains. Une politique municipale de la race, Paris, La Fabrique, 2014.

55. Pile S., "Introduction », op. cit.

56. Legros O., Olivera M., op. cit.

57. Scott J. C., La Domination et les arts de la résistance. Fragments du discours subalterne, Paris, Édition Amsterdam, 2009, p. 33.

58. De Certeau M., op. cit.

59. Dorier-Apprill E., Gervais-Lambony P. (dir.), Vies citadines, Paris, Belin, 2007. 
firmation, dynamique et ouvert à une redéfinition continue. Ce que nous nous attachons à défendre est que ces personnes considérées à la marge de la ville et de la société sont particulièrement citadines du fait de leur connaissance de l'espace urbain, de l'utilisation des ressources, de leur désir d'ancrage. Seule une reconnaissance profonde des capacités d'action de ces migrants, de leur interaction avec la ville, pourra permettre que d'autres futurs, loin des dystopies actuelles, soient imaginés. 